\title{
Secretory Immunoglobulin A: A Protective Factor in the Genital Mucosa
}

\author{
Paulo C. Giraldo ${ }^{1}$, Ana Katherine Gonçalves ${ }^{2}$ \\ and José Eleutério Junior ${ }^{3}$
}

\author{
${ }^{1}$ State University of Campinas - UNICAMP, Campinas, SP; \\ ${ }^{2}$ Federal University of Rio Grande do Norte; Natal, RN; ${ }^{3}$ Federal \\ University of Ceará; Fortaleza, CE; Brazil
}

\begin{abstract}
The genital mechanisms of defense are not well understood and are therefore ignored during therapy. This fact results in a great number of cases of treatment failure. The mucosa is an important protective factor of the genital female system, through self-defense mechanisms, and secretor antibodies (immunoglobulin A). The lymphoid tissue exerts protective anti-inflammatory activity, besides inhibiting microorganism adherence, neutralizes viruses and toxins and stabilizes the mucosal flora. Although certain microorganisms, such as viruses and fungus, are controlled by cellular immunity, secretory IgA can also exert an important role in the control of these agents. Key Words: Vaginal immunity, genital infections, HPV, Candida albicans, Secretory IgA.
\end{abstract}

During the last decades, the AIDS (Acquired Immunodeficiency syndrome) epidemic has induced us to recognize that mechanisms of defense of the genital tract affect the acquisition and evolution of genital infections. The importance of the host immune response in the control of genital infections was confirmed once we understood the mechanisms involved in the physiopathology of frequent infectious complaints in medical doctor's offices, such as: recurrent vulvovaginitis and HPV-induced lesions resistant to treatment. The genital tract has barriers that protect it against external microorganisms. These are mainly constituted by the skin and mucosal surfaces, which produce chemical substances, such as natural antibiotics (defensins), that hinder the adherence and the invasion of infectious agents into this epithelium [1-3]. When they surpass these obstacles, pathogenic microorganisms face various mechanisms of defense that include inflammatory phagocytes and antigen presenter cells that are set in motion in an attempt to eliminate the invaders [3]. During the induction of this initial mechanism of defense, activated leukocytes secrete cytokines and, along with the antigen presenter cells, invoke a specific immune response. This specific immune response can be humoral or cellular, depending on the type of microorganism that needs to be eradicated.

If there is there is a need to eliminate bacteria and extracellular parasites, antibody production (immunoglobulin) will occur constituting the humoral response [2,3]. Whenever viruses, fungi, bacteria and intracellular microorganisms need to be eliminated, activation of a cellular immune response [2,3] will occur. In the case of Candida albicans, as with all fungi, the most appropriate response for the eradication of infection

Received on 10 March 2006; revised 17 July 2006.

Address for correspondence: Dr. Paulo Cesar Giraldo. State University of Campinas. Alexander Fleming St, 848 13093-140 Campinas - SP, Brazil. Telephone - Fax: 55 (19) 3788-9306. E mail: giraldo@unicamp.br.

The Brazilian Journal of Infectious Diseases 2006;10(4):232-234. (C) 2006 by The Brazilian Journal of Infectious Diseases and Contexto Publishing. All rights reserved. is predominantly cellular. Recurrent candidiasis seems to occur due to a transitory defect of cellular immunity. An inadequate immunological response of the host to fungal infection is observed. The humoral immune response substitutes the cellular response, with exacerbated production of inefficacious antibodies against Candida [4,5]. On the other hand, recurrent candidiasis can denounce a severe deficiency of the immune system and can even be indicative of infection with HIV. Women with AIDS who have recurrent candidiasis die more precociously than those carriers of recurrent HIV without candidiasis [6].

Immunological deficiency influences the evolution of some genital infections. HIV induces immune depression, which modifies the natural history of HPV infection and propitiates oncogenesis, as well as other genital infections. HPV is more aggressive in HIV patients, with faster evolution of preneoplastic lesions to cancer. This is demonstrated by an increased frequency of infection in immunosuppressed individuals [7]. The association between HIV infection and progression of HPV-induced lesions strengthens this affirmation. The immunosuppression caused by HIV functions as a catalyst, favoring the evolution of pre-neoplastic lesions to cancer. Also, high-risk oncogenic HPV types interfere with the response of the immune system, subverting immunity and promoting viral persistence, another problem of relevance in the evolving process of this illness [8,9]. These and other types of evidence have demonstrated that the immune response of the host can be among the main factors that will define the natural history of genital infections [10]. Many studies still reveal the existence of a mechanism of defense inherent to the mucosa or lymphoid tissue associated with the mucosa (MALT) [11,12]. The MALT extends from the oral cavity to the gastrointestinal, respiratory and genitourinary tract. These mucosas present so many similarities that it is believed they are integrated. The evidence of a common mucosal immune system in human beings includes peripheral detention of antibodies of specific secretory immunoglobulin type A (IgAs) in various types of secretions, after oral exposition to microorganisms [13]. The MALT protects the organism against aggressors that penetrate into the mucosa, 
besides being the main producer of immunoglobulin (IgA). Most of the IgA is found in lymphoid tissues associated with the mucosa, although it is also detected in the blood in very small amounts [1].

The IgA that is released into the intestinal, respiratory and genitourinary mucosa, is known as secretory IgA (IgAs), because it conserves the fragment of the secretory component. IgAs is present mainly in secretions, such as saliva, colostrums, tracheobronchial secretions, milk, and in the epithelium of the genitourinary, gastrointestinal and respiratory tract; the main function of IgAs is to protect the lumen of these mucosa [1,2].

In healthy adults, the levels of IgAs are significantly higher than in children and the elderly [12-14]. Considering that secretion of IgA is originally derived from the mucosa, it is suggested that atrophy of the secretory mucosa could diminish the ability of older people to secrete IgA [15].

Patients with neoplasias, and immunosuppressed patients, also have lower levels of IgAs than healthy individuals, indicating that tissue alterations in the mucosa can harm the secretion of IgA, which would induce alterations in local immunity, resulting in a considerable increase in the infections in this mucosa [2,13-15].

The IgAs also exert a protective anti-inflammatory activity, suppressing the production of complement system mediators, inhibiting complement-dependent phagocytosis and the response of pro-inflammatory mediators, such as cytokines and arachidonic acid metabolites, coming from the phagocytic cells, thus preventing damage due to the inflammatory response. This anti-inflammatory property is important for maintaining mucosal integrity, functioning as a physical barrier between the host and pathogenic microorganisms [16-18].

The IgAs protects the mucosa in contact with microorganisms before they penetrate the lamina of the mucosa, forming an antigen-antibody complex, which is removed by the mucin secreted from the glandular tissue in various small sites of the mucosa $[3,16]$. Moreover, IgAs have been implied in the inhibition ofmicroorganism attack of the mucosal wall, removal of immunocomplexes and neutralization of viruses that infect cells, where antibodies usually offer little protection against intracellular pathogens. The IgAs can intercept viruses, interfering with their response and adherence, when the viruses go through infected epithelial cells [2,16-18].

In an important cross-sectional study, made of sex professionals in Kenya, it was observed that $75 \%$ of the women who were immunologically resistant to HIV had specific secretory IgA against the virus in the genital tract, while only $26 \%$ of the women infected by this virus had this antibody $[19,20]$. It was found, in further studies, those in uninfected women who are continuously exposed to HIV, the specific secretory IgA not only neutralizes the virus in secretions, but also induces transcytosis, in which this antibody blocks the action of the virus, interfering with its synthesis, besides neutralizing intracellular viruses [21,22].

In contrast to authors who consider only a dichotomous model (Th1/Th2) of immune response to explain the natural history of human papillomavirus infections [23,24], in which the cellular-mediated immunity determines the evolution of HPV-induced lesions, it was observed that it is possible to stimulate a humoral response to eradicate infection by HPV. It was also observed that parenteral injection of VLPs (virus like particles) induces the production of neutralizing antibodies, which confer protection against HPV infection [25-27]. An isolated cellular immune response does not seem to be the only host defense against HPV infection.

Recent studies have shown that patients with genital HPV presented lower salivary IgAs levels than the HPVnegative group [28]. The genital-HPV-positive women were more predisposed to present HPV in the oral cavity. In this same study, it was also observed that patients who presented concomitant oral and genital HPV, had still lower levels of IgAs than those who had only genital HPV [28]. It appears that these individuals had a secretory IgA deficiency, either due to intrinsic causes (systemic or local), or some mechanism induced by the virus presence.

It must be considered that some immunologicallycompetent women may present specific and transitory immunological deficiencies for particular infectious agents in the mucosa. Perhaps higher secretory IgA levels affect the initiation and progress of HPV infection. However, it is not known whether people infected with high-oncogenicrisk viruses who have an adjusted specific HPV immune response will have a more favorable evolution than those with low-risk HPV infection, but without an appropriate immune response. We believe that elucidation of the immunological mechanisms involved in the acquisition and progression of these infections will answer this and other questions.

\section{References}

1. Gorczynski R., Stanley J. Imunologia clínica. Reichmann, Rio de Janeiro; 2001.

2. Abbas A.K., Lichtman A.H., Pober J.S. Cellular and molecular immunology. 4a. ed., WB Saunders, California; 2003.

3. Riot I, Brostoff J, Male D. Imunologia. 6a. ed., São Paulo: Manole; 2003.

4. Witkin S.S., Hirsch J., Ledger W.J. A macrophage defect in women with recurrent Candida vaginitis and its reversal in vitro by prostaglandin inhibitors. Am Obstet Gynecol 1986;155: 790-5.

5. Witkin S.S. Immunologic factors influencing susceptibility to recurrent candidal vulvovaginites. Clin Obstet Gynecol 1991;34: 662-8.

6. Rhoads J.C., Wrign C., Reolfield R.R., Burke D.S. Chronic vaginal candidiasis in women with human immunodeficiency virus infection. JAMA 1987;257:3107-7.

7. Benton C., Shaidulah H., Hunter J.A. Human Papillomavirus in the immunosuppressed. Papillomavirus Rep 1992;3:23-6. 
8. Chirgwin K.D., Feldman J., Augenbraun M., et al. Incidence of venereal warts in human immunodeficiency virus-infected and uninfected women. J Infect Dis 1995;172(1):235-8.

9. Sedlacek T.V. Advances in the diagnosis and treatment of Human Papillomavirus infections. Clin Obstet Gynecol 1999; 42(2):206-20.

10. Scott M., Nakagawa M., Moscicki A.B. Cell-mediated immune response to Human Papillomavirus infection. Clin Diag Lab Immunol 2001;8(2):209-20.

11. Wan A.K., Seow W.K., Purdie D.M., et al. Immunoglobulins in saliva of preterm and full-term infants. Oral Microbiol. Immunol 2003;18(2):72-8.

12. Brandtzaeg P., Baekkevold E.S., Farstad I.M., et al. Regional specialization in the mucosal immune system: what happens in the microcompartments? Immunol Today 1999;20(3):141-51.

13. Jankovic J., Jelic C., Filipovic-Jeskovic I., Ristovic Z. Salivary immunoglobulins in cancer patients with chemotherapy related oral mucosa damage. Eur J Cancer Oral Oncol 1995;31B(3):160-5.

14. Tappuni A.R., Challacombe S.J. A comparison of salivary immunoglobulin A (IgA) and IgA subclass concentrations in predentate and dentate children and adults. Oral Microbiol. Immunol 1994;9(3):142-5.

15. Ventura M.T. Evaluation of IgA1-IgA2 levels in serum and saliva of young and elderly people. Allergol. Immunopathol 1991; 19(5):183-5.

16. Russell M.W., Mestecky J. Humoral immune responses to microbial infections in the genital tract. Microbes Infect 2002;4(6):667-77.

17. Russell M.W. IgA as an anti-inflammatory regulator of immunity. Oral Dis 1999;5:55-67.

18. Egmond M.V., Damen C.A., Spriel A.B.V., et al. IgA and IgA Fc receptor. Trends Immumol 2001;22(4):205-11.
19. Kaul R., Trabattoni D., Bwayo J.J., et al. HIV-1-specific mucosal IgA in a cohort of HIV-1-resistant Kenyan sex workers. AIDS 1999;4(1):23-9.

20. Kaul R., Plummer F.A., Clerici M., et al. Mucosal IgA in exposed, uninfected subjects: evidence for a role in protection against HIV infection. AIDS 2001;15(3):431-2

21. Devito C., Hinkula J., Kaul R., et al. Mucosal and plasma Iga from HIV-exposed seronegative individuals neutralize a primary HIV1 isolate. AIDS 2000A;8(13):1917-20.

22. Devito C., Broliden K., Kaul R., et al. Mucosal and plasma iga from hiv-1-exposed uninfected individuals inhibit hiv-1 transcytosis across human epithelial cells. J Immunol 2000B;1(9):5170-6

23. Grassegger A., Rollinger-Holzinger I., Zelger B.W.H., et al. Spontaneous or interferon-g-induced t-cell infiltration, hla-dr and icam-1 expression in genitoanal warts are associated with th1 or mixed th1/th2 cytokine mrna expression profiles. Arch Dermat Res 1997;289:243-50.

24. Clerici M., Merola M., Ferrario E., et al. Cytokine production patterns in cervical. Intraepithelial. Neoplasia: association with human papillomavirus infection. J Natl Cancer Inst 1997;89:245-50.

25. Tartour E., Gey A., Sastre-Garau X., et al. prognostic value of intratumoral vaginal interferon gamma messenger rna expression in invasive cervical carcinomas. J Natl Cancer Inst 1998;90:287-94.

26. Thapar M.A., Parr E.L., Parr M.B. Secretory immune responses in mouse vaginal fluid after pelvic, parenteral or vaginal immunization. Immunol 1990;70:121-25.

27. Balmelli C., Roden R., Potts A., et al. Nasal immunization of mice with human papillomavirus type 16 virus-like particles elicits neutralizing antibodies in mucosal secretions. J. Virol 1998;72(10):8220-29.

28. Gonçalves A.K.S., Giraldo P., Barros-Mazon S., et al. Secretory immunoglobulin A. Eur J Obstet Gynecol Reprod Biol 2006;Feb124(2):227-31. 